\title{
Polysèmes
}

Revue d'études intertextuelles et intermédiales

\section{Une folle liberté que je dois maîtriser pour me dire : endochronies du journal intime}

\section{Philippe Amen}

\section{(2) OpenEdition}

\section{Journals}

Édition électronique

URL : http://journals.openedition.org/polysemes/2011

DOI : 10.4000/polysemes.2011

ISSN : 2496-4212

Éditeur

SAIT

Référence électronique

Philippe Amen, «Une folle liberté que je dois maîtriser pour me dire : endochronies du journal intime », Polysèmes [En ligne], 17 | 2017, mis en ligne le 30 avril 2017, consulté le 01 mai 2019. URL : http:// journals.openedition.org/polysemes/2011 ; DOI : 10.4000/polysemes.2011

Ce document a été généré automatiquement le 1 mai 2019.

Polysèmes 


\title{
Une folle liberté que je dois maîtriser pour me dire : endochronies du journal intime
}

\author{
Philippe Amen
}

1 Parler de soi au plus profond de l'intime, construire une parole de soi. C'est une tentation qui hante la littérature à des degrés divers, à des degrés qui sont autant de temporalités expérimentales et instinctives. Le récit de vie, qui se décline génétiquement en cinq formes (les mémoires, l'autobiographie, le roman autobiographique, l'autofiction et le journal intime), offre à n'en pas douter une lecture du temps particulière qui s'adapte à l'homme et à son dessein. Le mémorialiste s'inscrit dans l'Histoire, c'est-à-dire un temps qui dépasse son existence, l'autobiographe se souvient, étape après étape, de sa vie, l'adepte du roman autobiographique, le concepteur d'autofiction fondent un projet d'écriture, le diariste, lui, ne fait que réagir au plus petit dénominateur commun d'un temps qui fait sens en nous: la journée. On se rend compte que la parole autobiographique a le choix de sa temporalité : de l'échelle de l'Histoire à l'échelle de ma journée, il y a un monde! Si l'on voulait d'ailleurs élargir cette catégorisation en cinq formes à une pratique très contemporaine d'expression de soi, certes peu introspective mais néanmoins garante d'une trace de soi en écriture, on peut faire remarquer que l'usage de Twitter ou de Facebook, aujourd'hui, réduit cette échelle de la journée à celle de la minute, à celle d'une pluie de secondes. Mon propos liminaire a pour objectif de rappeler ces différences de structures pour fixer le champ d'occupation du temps par le rédacteur d'un journal intime: matériellement, le diariste s'occupe d'un temps borné entre le lever du soleil et le coucher du soleil.

2 Cette structure matérielle qui offre un rendez-vous, un rythme, une profération, c'est une structure fortement humaine. Mais si l'on comprend que le besoin d'écrire son journal se coule dans cette unité de mesure, on ne peut cependant pas s'arrêter là. Il nous faut penser trois spécificités temporelles de ce type d'écrit, l'une qui a trait à un invariant ontologique, une deuxième qui peut éclairer une posture morale et enfin une dernière qui 
met en lumière une absence de modélisation textuelle. La première spécificité s'appuie sur la succession des jours qui forme masse, qui dessine, cahier après cahier, une présence scripturale. Or cette présence qui se dit par le présent est indéterminée dans l'avenir : la finitude ne se pose généralement pas et le diariste n'écrit pas un texte qui a pour objectif une fin, une apothéose, un art. Dans le champ de la réception, nous, lecteurs de journaux intimes, nous savons qu'entrer dans une vie par effraction, par la lecture de ces journées successives, ne va pas nous amener à une fin rhétoriquement fermée. Le diariste vit, me semble-t-il, à la fois un inconfort et une jubilation métaphysiques qui disent ceci de très clair : un journal intime comme une vie ne se termine pas. En ce sens qu'il ne se soumet pas à un arrangement de choses dans une durée déterminée. Les diaristes qui doublent leur existence par cette narration quotidienne (par exemple le cas du philosophe suisse Henri-Frédéric Amiel et ses 16800 pages de journal intime sur lequel je vais fonder une grande partie de mon analyse) s'exercent à une écriture du détail, parce qu'il n'y a pas de limite et pas d'achèvement, à un ressassement ouvert. Certes c'est moins le cas dans l'écriture de textes modestes : le journal adolescent de l'écrivain Pierre Louÿs par exemple, journal qu'il tient entre le 24 juin 1887 et le 16 mai 1888, se termine par ces mots : «Et maintenant je dis adieu à ce premier cahier qui renferme mes secrets les plus chers, à qui je me suis confié tant de fois, quand j'avais besoin de m'épancher, et que j'ai tant de plaisir à feuilleter déjà, pour penser un peu en arrière et revivre ma vie passée » (284). Si cet adieu est solennel et surdéterminé, nous remarquons qu'il est contredit par l'expression « ce premier cahier » et par le plaisir de la relecture, pratique habituelle du diariste ${ }^{1}$ lié à la pensée de l'éternel recommencement. La seconde spécificité est un habitus moral. Le bilan de la journée entraîne le fameux examen de conscience encouragé par l'Église pendant tout le XIX ${ }^{e}$ siècle, siècle de la confession intime comme mode sociale. Michel Foucault, définissant l'origine de cette sommation judiciaire, indique, dans l'un de ses cours au Collège de France ${ }^{2}$, qu'il s'agit de ne pas occulter le fondement pythagoricien de l'examen de conscience résumé par le précepte : «Préparetoi à un doux sommeil en examinant tout ce que tu as fait dans la journée » (460). Foucault insiste sur cette pratique comme étant celle d'une relecture purificatrice de nos actes juste achevés en vue d'un sommeil sans agitation. Par conséquent, il fonde la pertinence de ce besoin non pas sur une tension morale qui met au cœur de ses volontés un dispositif de tribunal mais bien une dichotomie purement temporelle qui permet de recommencer (donc de revivre en pensée) et de poursuivre (donc de dormir). Très rapidement, il semble que les stoïciens pervertiront ce simple constat rythmique pour en faire une enquête comptable, un examen des fautes que le christianisme médiéval reprendra à son compte, bien évidemment. La troisième caractéristique est liée au fondement secret du journal, que son auto-destination même sacralise. Quand bien même la circulation des textes et des pensées s'y donne à lire, j'ai tendance à croire que les balbutiements de cette forme littéraire, au XIX ${ }^{\mathrm{e}}$ siècle, s'accompagnent d'une crispation auctoriale due à une impossible postérité. Cependant, pour compenser cet empêchement, l'écrit intime dispose d'une liberté totale du dire et de l'écrire. Car on peut tout se permettre dans ce huis-clos: dessiner des croquis dans la marge comme le fait Stendhal, mélanger les langues comme le pratique Valery Larbaud, faire la liste sur une échelle temporelle de ses influences littéraires, ce que fait Queneau, ou coder ses pensées inavouables, comme chez Benjamin Constant, parce que ce dernier craint la matérialisation du secret. Si l'on ajoute que l'élan introspectif peut se débarrasser sans vergogne du souci orthographique, modélisation textuelle par excellence, on en vient à 
penser que le cahier intime s'occupe davantage d'une vivante singularité que d'une normativité littéraire.

3 En guise de premier bilan, on peut se demander sous quels marqueurs temporels la parole du diariste peut exister. Je proposerai donc de dire que le diariste accède à un vertige, à une folie du temps, qui a les caractéristiques que je viens d'évoquer: un champ d'investigation sans finitude, autrement dit un inachèvement; un apprentissage de l'éternel recommencement; une liberté face à ce présent secret qui s'exprime à tout moment dans la façon de le fixer. Le diariste aurait-il alors inventé le seul «genre littéraire » libre de toute norme, un réceptacle d'extravagances et de déconstructions, un Fay ce que vouldras textuel et existentiel?

4 Force est de constater que la réponse est décevante. C'est sans doute un paradoxe de découvrir que pour être au plus près de la vie mouvante des journées qui passent, le diariste a besoin de multiplier les conduites normatives liées à un contrôle du temps et donc du réel. Peut-être angoissé par la liberté de dire le mouvement, il préfère se glisser dans un carcan, un rituel, une monomanie. Il construit dans un temps personnel et répercuté la stabilité de son moi. Pour éclairer ce besoin, il convient de classer ces conduites par de simples affirmations.

J'en pose ici trois.

\section{La datation est un défi}

6 Les journaux intimes régulièrement tenus et qui couvrent une grande partie de la vie de leur auteur proposent une datation stricte qui fonde la chronologie. Il faut évidemment faire la distinction entre le moment de l'écriture (Amiel saisit toujours l'heure de son bilan) et le moment narré plus ou moins minutieusement découpé. Le cas le plus saisissant d'une fragmentation, d'une miniaturisation de la journée est le journal d'Arthur Schnitzler (il s'étend de 1879 à 1931) qui aimait fractionner la narration de la journée en quatre temps immuables. Avec une précision horlogère, il empile ces quatre moments indexés par des abréviations : matin, après-midi, dîner, après le dîner. Cette partition donne à lire un désir de remplir le vide et fonde le fantasme de la durée du jour comme unité parfaite. C'est un temps où tout doit se passer, où les heures se métamorphosent en événements sans quoi on perd son temps, sans quoi le temps n'est plus addition mais soustraction. Astucieusement, Amiel écrit en 1861 : «Je vis non seulement au jour le jour, mais sans unité intérieure même d'un jour " (IV, 105), ce qui signale chez lui un déchirement temporel intéressant: l'homme universel qui se construit dans le temps social et qui a besoin de grands projets (par exemple l'inscription dans une continuité historique) et l'homme intérieur qui se mesure à la journée remplie. Son autodénigrement ici révèle qu'il est mécontent de cette double inscription temporelle. On sent bien ce que le diariste souhaite révéler : le temps produit un sens, et comme l'écriture au plus près du temps est capable de le fixer, le temps devient sens. Nous devons nuancer. Dans Différence et Répétition, Gilles Deleuze oppose fondation du temps et fondement du temps, l'une occupée par la mouvance du présent qui se ceint d'habitudes, l'autre réfléchissant sur le passé pur : la fondation occupe le sol (c'est l'usage, l'utile, le fertile, le futile), le fondement vient du ciel (c'est la mémoire). Ce temps du bas et ce temps du haut n'ont pas la même logique et c'est en somme, pour chaque être humain, un véritable défi de faire coïncider leur chronologie propre. Reste que le jugement sur la journée, son efficience d'usage ou de mémoire, hante le diariste. Le pessimisme d'Amiel lui fait noter 
dans l'un de ses cahiers que sa journée «est supprimée du calendrier de la vie active » (III, 729), dans un autre qu'il a vécu « une journée si mauvaise qu'on se demande combien il en faudrait de ce type pour en valoir une bonne » (III, 193), et dans un autre encore « une journée qui n'a servi qu'au vieillissement» (IV, 487). L'historien anglais Edward Gibbon, dans son Journal écrit à Lausanne, va jusqu'à fonder un principe d'écriture sur ce genre de constatation : «Est-il nécessaire de parler d'un jour qui n'a pas existé ?» (32). Il différencie donc les jours vivants et les jours morts. Ce jugement sur la valeur de notre engagement dans l'ordre du vivant est un topos de notre psychologie. Mais la réaction de Gibbon est assez rare chez les diaristes qui ont plutôt l'habitude de combler le temps mort. Récriminations, analyse morale, résolutions réactivées : tout un attirail de langage programmatique vient à la rescousse de la déception et du vide. N'oublions pas que les diaristes réguliers sont majoritairement des hommes et des femmes solitaires pour qui le corps social offre un étrange jeu de dupe. Ils font davantage confiance à la page blanche de leur cahier qu'à l'échange et à l'altérité. Les historiens peuvent facilement démontrer, comme l'écrit John E. Jackson, que «l'essor de la recherche ou de l'affirmation de soi appartient aux caractéristiques d'une société qui a vu, dans le même temps, l'atomisation de ses membres s'accentuer toujours davantage » (59). Cependant l'art rhétorique, ici la captation du ferment fictionnel de la vie, réduit ce sentiment d'abandon et d'isolement. On sait bien qu'écrire c'est ne jamais être seul! D'autant que, dans cet enfermement dans la chronologie, existe une petite vanité intéressante. Le défi qui consiste à transformer une vie en un agrégat de moments datés fait la part belle au fantasme de l'empilement, à la symbolique de la superposition. La date c'est l'instant. Ces instants se suivent et forment une histoire de vie. Cette histoire est répercutée par un support. Le support c'est le cahier. Le nombre de cahiers griffonnés équivaut à la durée de ma vie. Il y a donc adéquation profonde entre la matière de la fixation et la concrétisation du temps qui passe. D'où la numérotation précise des cahiers, une rigueur de présentation, parfois une indexation. Le nombre de cahiers est une doublure de la datation et elle signale un empilement glorieux. Une preuve de l'existence du moi. Elle dit aussi une continuité profonde de cette écriture, qu'on pourrait penser - à tort!- être une écriture fragmentaire alors qu'elle se fonde sur un besoin d'unification et de mémoire.

\section{Exister c'est calculer}

7 Nous avons vu que l'examen de conscience s'était rapidement tourné, dans notre histoire, vers le redressement moral. Or, pour s'évaluer, il faut créer des outils et des automatismes. J'ai toujours été frappé par deux adjectifs qu'utilise Stendhal dans son Journal de 1810 : il dit vouloir restituer " les détails mathématiques et sévères destinés à présenter le procès-verbal de [s]a manière d'être » (972). Mathématique et sévère. Tout est dit. La rigueur du calcul et le regard du juge. L'ordonnance mathématique, l'emploi du temps et le jugement moral se confondent souvent pour créer des bilans désagréables. L'écrit personnel devient le moyen rationnel de comptabiliser les heures et de calculer les dérives, comme s'il s'agissait de mettre en forme un diagramme thérapeutique. Il convient ici de faire référence à la figure de Marc-Antoine Jullien (1775-1848), le fameux auteur de deux ouvrages qui font date dans la conscience domestique de l'éducation par le temps : l'Essai sur l'emploi du temps ou méthode qui a pour objet de bien régler sa vie, premier moyen d'être heureux, destinée spécialement à l'usage des jeunes gens de 15 à 25 ans (qu'on nomme aussi «Mémorial horaire », et datant de 1810) et sa Lettre à la nation anglaise, sur 
l'union des peuples et la civilisation comparée, sur l'instrument économique du temps, appelé biomètre, ou montre morale (publiée en 1833). Grâce à lui, la première génération des diaristes du XIX ${ }^{e}$ siècle (dont Maine de Biran et Amiel) prend connaissance de ce système : " une suite de petites tables composées de colonnes, représentant tous les emplois possibles de la vie humaine et sociale et tous les rapports qu'elle embrasse. Chaque table contient dix lignes pour dix jours et une dernière ligne de récapitulation $»^{3}$. Ce dispositif peut avec le recul nous apparaître futile ou savoureux mais il existe pour réglementer notre horloge intérieure et nos humeurs, et fournir par ses calculs le moyen de progresser. Rappelons que l'inventeur de cette micro-analyse temporelle est aussi le créateur d'un syntagme, sinon d'un concept, fort moderne : celui de science de l'éducation. Parce qu'elle intériorise l'expérience de l'inachèvement, l'écriture intime multiplie les approches quantitatives. Une journée doit être utile, tournée vers le travail, pense Amiel : « le chiffre à adopter c'est 8 heures au minimum, 12 au maximum, 10 en moyenne » (II, 1245). Il établit des rapports entre l'état physique et la production intellectuelle: "l'heure de 3h après-midi est mauvaise pour la tête » (IV, 139) mais on est rassuré par le fait que «les heures les plus propres à la phénoménologie sont celles qui précèdent l'aube » (II, 422). Se met en place, on le sent, un temps personnel propre aux obsessions des diaristes, un temps incommunicable parce qu'il oscille toujours entre l'expérience et la généralisation. Dans la mise en place d'un programme moral, Amiel calcule tout: sa vitesse de lecture, l'ampleur d'une migraine, les chances d'un mariage. La minutie avec laquelle un diariste calcule ses désirs ou ses désagréments peut surprendre. Nous trouvons dans les cahiers du diariste suisse deux étonnantes remarques, toutes deux de 1866. La première concerne l'obsession amiélienne de tomber amoureux ou plutôt de trouver la femme idéale. Il note : « La femme nue est belle une fois sur vingt, et trois ans sur soixante et dix. C'est-à-dire qu'il y a 470 à parier contre un, qu'en photographiant une femme sans voile, on fait une indécence sans arriver à un effet esthétique " (VI, 492). Voilà qui est dit. La seconde est la narration d'une contrariété vraiment anodine : «J'ai passé une nuit blanche, grâce au chien d'un voisin qui n'a pas discontinué d'aboyer de $2 \mathrm{~h} 1 / 2$ du matin à $7 \mathrm{~h} 1 / 2$; cela sous la fenêtre de ma chambre à coucher. Impossible de fermer l'œil. J'ai compté avec ma montre le nombre de jappements pendant une certaine durée. Il s'en suit que ce jeune chien a hurlé 25000 fois, dans une cour étroite qui résonne comme un portevoix » (VI, 105). Le temps du calcul est si intégré à l'esprit d'unification du moi dans l'événement qui survient, à ce moi fixateur d'un réel quantitatif et normatif, qu'il paraît absurde et vain dans un autre contexte. La démonstration peut en être faite dans le rapprochement entre deux notations chez Amiel. La première, nous venons de la citer : les 25000 aboiements du jeune chien. Comparons cette temporalité vécue avec une deuxième notation lorsqu'il lit le Journal de Louis XVI. Voici son jugement : « Le journal de Louis XVI, publié par Nicolardot, témoigne d'une bêtise ultra-bourbonnienne. Ce pauvre sire compte les bêtes qu'il a tuées (jusqu'à 200 hirondelles en un jour) et les révérences des courtisans, mais ne voit, n'entend et ne comprend rien autour de lui. C'est un Nemrod stupide auquel la décapitation a fait une auréole. Il a occis en 13 ans 1300 cerfs et 180000 pièces de gibier mais il n'a pas eu une pensée » (IX, 636). Cet écho dans la minutie des deux scripteurs, qu'Amiel se montre incapable d'identifier pour son propre cas, renforce, me semble-t-il, l'enfermement aveugle dans une ritualisation qui, si elle ne semble pas immédiatement sienne, a toutes les chances d'être jugée ridicule. Bien évidemment, sur l'échelle du temps, les calculs que nous trouvons dans ces journaux intimes auto-destinés concernent tout aussi bien un passé revisité, un présent comptabilisé qu'un avenir en espérance. Il existe des élans prédictifs (nommer la date de 
sa propre mort par exemple) mais aussi une manière de s'inclure dans l'angoissante condition humaine par un changement d'échelle. Le poète et traducteur Armel Guerne écrit dans son Journal de 1942 : «J'ai connu jusqu'ici onze mille et quelques cents jours. J'ai donc échappé à la mort, inexplicablement, onze mille et des centaines de fois » (46). De même, Amiel a le même genre de calcul : «Tu as repoussé 15425 fois la mort, par le sommeil et la nourriture » (VI, 595), avoue-t-il en 1866.

\section{Si le temps accélère, je me redéfinis}

Quand les drames collectifs s'immiscent dans l'écriture la plus personnelle, que devient cette monomanie, ce goût de la maîtrise, ces réactions qu'on pourrait croire sans consistance? Mon dernier point portera sur le sentiment psychologique d'un emballement de l'Histoire que je voudrais rapidement illustrer par le Journal d'Hélène Berr tenu entre 1942 et 1944. Sa publication tardive (2008) nous a fait connaître le destin de cette jeune Parisienne juive qui terminera tragiquement sa vie au camp de BergenBelsen. Le manuscrit d'Hélène Berr débute comme le journal typique d'une jeune intellectuelle inscrite à la Sorbonne qui note avec sensibilité les émois de sa jeunesse entre amitiés et amours débutantes. Profondément auto-destiné, ce journal a les caractéristiques d'un huis-clos serein et lumineux, habité par cet inachèvement temporel dont nous avons parlé. En somme, pour reprendre l'expression de Philippe Lejeune, elle produit un texte « virtuellement infinissable dès le départ » (Viollet 211). Hélène Berr, par exemple, ne se considère pas comme quelqu'un qui écrit, parce que, explique-t-elle, " pour moi, peut-être à tort, écrire implique un dédoublement de la personnalité, sans doute une perte de spontanéité, une abdication » (184). Nous y trouvons trace de ces détails qui font que la temporalité (rythme, datations, ressassements, anniversaires) est vécue comme une affaire strictement personnelle. Et puis le texte se coupe en deux. Cette césure est due aux événements qui s'enchaînent dans le Paris occupé de cette période, au bouleversement intime que l'on découvre dans ses réactions progressives aux lois antijuives. L'écriture bascule dans le témoignage. En quelques mois, la perspective, dans l'échelle de la mémoire du vivant, a radicalement changé : «Je sais pourquoi j'écris ce journal, je sais que je veux qu'on le donne à Jean si je ne suis pas là lorsqu'il reviendra. Je ne veux pas disparaître sans qu'il sache tout ce que j'ai pensé pendant son absence, ou du moins une partie. Car je "pense" sans arrêt. C'est même une des découvertes que j'ai faites, que cette conscience perpétuelle où je suis » (206). On pèsera sans doute avec profit la différence qu'un diariste peut définir entre une absence de finitude et une conscience perpétuelle. Sartre, qui s'est toujours tenu à l'écart de l'épanchement quotidien et qui juge avec sévérité, selon son expression, "les maniaques de l'analyse type Amiel», a pourtant éprouvé le besoin de tenir ses Carnets de la drôle de guerre. Mais l'ossature du travail se fonde sur l'écriture comme trace "historique». Sartre se veut avant tout créateur. Répondant à cette difficulté qui veut qu'il se cache toujours dans l'un de ses personnages romanesques, Sartre a cette phrase qui, en effet, porte l'étendard de l'antidiariste : «La différence essentielle entre Antoine Roquentin et moi, c'est que moi j'écris l'histoire d'Antoine Roquentin " (410). Rien en Sartre ne peut aboutir à avouer que son moi est définissable par l'angoissante fuite du temps. En revanche, le diariste, tourné vers une activité de redondance sans création, vers une approche quasi tragique, racinienne, de l'expression intime, façonné qu'il est par cette journée du lever du soleil au coucher du soleil, ne souhaite qu'une chose : répercuter un peu de soi dans cette structure mesurée. 
9 À part quelques basculements de perspective, le diariste qui double sa vie par un empilement de cahiers, dans un mouvement spontané et concurrent, plonge dans une recherche d'exactitude qui lui permet, selon la formule de Paul Valéry, de « sténographier l'instant» (I, 569). Ce qui se joue, dans ces textes personnels voire secrets, c'est un langage profondément lié à ces dispositifs de traduction d'un temps intérieur sans cesse convoqué. Pour les définir, pour en souligner la singularité en même temps que la nécessité inédite, on doit se résoudre à fonder un néologisme barbare : les journaux intimes sont remplis d'endochronies, traces d'une temporalité employée par le seul moi du scripteur, d'un temps auto-suffisant, d'un temps qui rompt avec celui du corps social, qu'il agisse dans le fractionnement de ses unités ou qu'il s'ouvre à des élans plus vastes, qu'il illustre un effort de lucidité morale ou un impressionnisme des actes, des pensées et des sentiments.

10 Une endochronie serait donc un temps investi par le moi, lui-même investi par le temps intime. Même si on peut l'entendre comme un concept à forme gigogne en cela qu'une endochronie serait un temps plus petit logé dans un temps infini et social, il me semble qu'il peut s'agir, pourtant, tout à la fois, d'une sensibilité extrême dans la vertu de l'immédiateté qui fonde une possession (mon écriture est une collecte de mon existence dans le temps collectif qui sert mon écriture) et d'un goût de la structuration permettant de fermer le temps au temps, d'en borner l'étendue (je ne fais vivre que le temps que j'écris, je le fais exister dans la seule dimension de mon processus, je le contrains). Fondation (possession) et fondement (à ciel fermé), pour reprendre les idées de Deleuze. Il y a contiguïté des deux structures ${ }^{4}$. Si bien que le diariste crée une tension, fonde ainsi un troisième temps. Un tiers temps de l'intime-écrit. Ce cocon rassurant, ce réflexe qui soumet la liberté discursive à son contraire disent bien que la chronographie maniaque réagit effectivement à l'immensité de nos vies durables, mais elle crée surtout une volonté de dépassement. Cette tension entre le temps des calendriers, le temps biologique et social, et celui des préoccupations répercutées de sa journée oblige le diariste à inventer une troisième voie temporelle. Une voie qui aurait pour outils le temps spatialisé et le temps intériorisé de Bergson dans la perspective instinctive d'écrire une facticité autonome. Dès lors, ce sont bien les faits écrits, gardés dans une boîte à mémoire, qui dominent la réaction temporelle: "Nulla dies sine linea " était la devise d'Amiel qui pensait, comme nombre de ses congénères, qu'écrire équivalait à vivre, et que ce temps n'était pas perdu. Pourtant, voilà une occupation qui rend perplexe, si immédiate et parfois sans valeur, si instinctive et profondément obsessionnelle. Pourtant, voilà une occupation analytique qui approche une vérité des comportements et des relations, fortement essentielle dans la perception des jeux de conscience. Maurice Blanchot avait pressenti cette particularité de l'intime-écrit, ce saut dans l'endochronie si manifestement autre. Il écrivait dans Le Journal intime et le récit : « On écrit pour sauver les jours, mais on confie son salut à l'écriture qui altère le jour » (256). Le verbe altérer, si soigneusement choisi, donne l'idée d'une modification qualitative, d'une transformation ou d'une dégradation. Par ce simple verbe, il certifie qu'existe ici une différence de nature. Celle-ci ne constitue pas une pose littéraire ${ }^{5}$ car le journal intime des origines, auto-destiné, dépositaire d'une âme inquiète, est un texte sans référence et sans postérité, qui se plaint et se cherche, qui ressasse et calcule mais qui s'empare, inconsciemment d'une temporalité inhérente à cet enfermement dans une régularité du dire. On tient là, peut-être, la seule forme littéraire qui invente son propre temps, un temps modeste, entassé, acharné, extravagant. 
11 D'Amiel qui veut simplement « accoucher de lui-même » à Hélène Berr qui symbolise le cri d'une humanité aux abois, chaque diariste, sans doute, conçoit ce temps d'écriture comme une déposition, une trace créative qui a pour objet le Temps lui-même. Même dans les exemples de journaux où la folie entre en jeu (on peut penser aux Carnets secrets de Louis II de Bavière), le texte invente sa propre norme. Une endochronie ne présente donc pas, du point de vue de son résultat, un univers subi, le texte qui se lit semble au contraire, parce que justement la soumission à la régularité est déjà là, une autosuffisance active et créative, même si elle est, comme nous l'avons vu pour Hélène Berr, une pensée après coup. Amiel croit souvent que l'écriture de ses cahiers intimes remplace et désactive l'élan de l'ambitieux, du conquérant, mais au détour d'une plainte, il est bien obligé d'avouer : ce journal « m'a aidé à penser, et il me prouve que j'ai réellement vécu » (1081). Et l'on revient à la double dimension de jugement et de preuve que détient ce réceptacle temporel.

Résumons donc notre hypothèse : ce temps particulier se construit avec, contre et à l'écart du temps d'autrui, pour faire exister un réel devenu texte, une journée devenue quelques lignes de plus (une quantité non négligeable), et aussi, et par un ricochet très compréhensible dans cette auto-destination sacrée, pour créer l'existence du scripteur. Ainsi, il multiplie les indices d'un fonctionnement cadenassé, obsessionnel et profondément intime.

Reste à savoir si ces endochronies, ces signes de l'intime-écrit, donnent à lire une sagesse ou une folie. La réponse tient peut-être dans la façon toute personnelle dont chacun peut réagir à quelques aboiements de chien!

\section{BIBLIOGRAPHIE}

Amiel, Henri-Frédéric. Journal intime. Lausanne : L’Âge d'Homme, 1976-1994.

Berr, Hélène. Journal. Paris : Éditions du Seuil, « Points », 2009.

Blanchot, Maurice. Le Livre à venir. Paris : Gallimard, « Folio Essais », 1959.

Braud, Michel. La Forme des jours. Pour une poétique du journal personnel. Paris : Éditions du Seuil, 2006.

Foucault, Michel. L'Herméneutique du sujet : cours au Collège de France, 1981-1982. Paris : Gallimard, 2001.

Guerne, Armel. Journal 1941-1942. Lectoure : Le Capucin, 2000.

Jackson, John E. Passions du sujet. Essais sur les rapports entre psychanalyse et littérature. Paris :

Mercure de France, 1990.

Le Journal de Gibbon écrit à Lausanne. Lausanne : Librairie de l'Université de Lausanne, Rouge et Cie, 1945.

Lejeune, Philippe et Catherine Viollet (dir.). Genèses $d u$ "je », Manuscrits et autobiographie. Paris : CNRS Éditions, 2000. 
Jullien, Marc-Antoine. Lettre à la nation anglaise, sur l'union des peuples et la civilisation comparée, sur l'instrument économique du temps, appelé biomètre, ou montre morale. London: Bossange, Barthès and Lowell, 1833.

Louÿs, Pierre. Mon Journal. Paris : Éditions du Seuil, 1994.

Sartre, Jean-Paul. Carnets de la drôle de guerre. XIV. Paris : Gallimard, 1995.

Stendhal. Journal. Paris : Gallimard, « Bibliothèque de la Pléiade », 1955.

Valéry, Paul. Cuvres. Paris : Gallimard, « Bibliothèque de la Pléiade », tome I, 1957.

\section{NOTES}

1. L'originalité du journal de Louÿs est en effet que sa relecture a engendré un regard critique puisqu'il annota ses propres propos à de nombreuses reprises, permettant ainsi un télescopage des temps.

2. Cours du 24 mars 1982 in Michel Foucault, L'Herméneutique du sujet, Paris : Gallimard, 2001.

3. Marc-Antoine Jullien, Lettre à la nation anglaise, sur l'union des peuples et la civilisation comparée, sur l'instrument économique du temps, appelé biomètre, ou montre morale, London: Bossange, Barthès and Lowell, 1833, 15. Notons que nous sommes encore chez Jullien dans un modèle temporel décimal issu de la Révolution française.

4. Michel Braud note qu'il peut y avoir coïncidence. Le diariste, dit-il, « manifeste la coïncidence de l'écriture et du mouvement temporel du monde, par la conscience qui saisit le monde et conduit à l'écriture " in Michel Braud, La Forme des jours. Pour une poétique du journal personnel, Paris : Éditions du Seuil, 2006, 129.

5. Pose littéraire que l'entrée du journal intime comme genre éditorial génèrera, peu ou prou après Gide.

\section{RÉSUMÉS}

Le journal intime, pratique textuelle auto-destinée, installée dans le champ social du XIX ${ }^{\mathrm{e}}$ siècle, pourrait donner lieu à l'expérimentation d'une écriture totalement libérée. Or, quand on examine le rapport à la temporalité des diaristes, on s'aperçoit que l'enjeu de ces textes est de fixer une ritualisation et une normativité. Cette étude veut montrer comment, par la datation, les calculs obsessionnels et la recherche d'un sens, s'élabore un temps auto-suffisant. Nous voulons nommer « endochronie » cet usage créatif du temps à l'intérieur d'un texte sans référence et sans postérité. L'œuvre d'Henri-Frédéric Amiel ou, au XXe siècle, le journal d'Hélène Berr sont les textes principaux qui permettent ici de définir la notion d'endochronie.

One might think that the self-destined literary practice of diary-keeping, which used to be a popular nineteenth-century social practice, could produce wholly liberated experimental works. However, when one ponders the diarists' approach to temporality, one realises that their texts are apt to build up rituals and set up norms. This paper aims to show how a self-reliant normative temporality is established by means of dates, obsessive calculations and quests for meaning. Hence, the paper defines the idea of "endochrony", i.e. the creative use of time within 
the text without any reference nor any bid for posterity. The works of Henri-Frédéric Amiel or the twentieth-century literary diary written by Hélène Berr are good instances of the phenomenon of endochrony.

INDEX

Mots-clés : journal intime, temporalité, endochronie, auto-destination, XIXe siècle Keywords : diary, temporality, endochrony, self-destined texts, 19 th century

\section{AUTEURS}

\section{PHILIPPE AMEN}

Philippe Amen est spécialiste du journal intime au XIX ${ }^{\mathrm{e}}$ siècle et notamment d'Henri-Frédéric Amiel. Il est l'auteur de plusieurs études sur le sujet dans des volumes d'histoire de la littérature aux éditions PUF et Payot Lausanne. Il est chargé de cours à l'Université de Poitiers. 